\title{
AS PRODUÇÕES MATEMÁTICAS DE LORENZO MASCHERONI COMO FONTE PARA PRODUÇÃO DE MATERIAIS EDUCACIONAIS
}

\section{LORENZO MASCHERONI'S MATHEMATICAL PRODUCTION AS A SOURCE FOR THE PRODUCTION OF EDUCATIONAL MATERIALS}

\author{
José Damião Souza de Oliveira ${ }^{1}$ \\ Secretaria de Estado da Educação e da Cultura - SEEC \\ Giselle Costa de Sousa ${ }^{2}$ \\ Universidade Federal do Rio Grande do Norte - UFRN
}

\section{Resumo}

Neste trabalho temos o intuito de expor parte dos resultados de uma pesquisa, em que fizemos um estudo histórico bibliográfico das obras produzidas por um matemático e poeta italiano do século XVIII, a saber Lorenzo Mascheroni (1750 - 1800). Para tanto, nos apoiamos em elementos da pesquisa qualitativa do tipo bibliográfica, assim como, nos valemos de seus instrumentos e procedimentos. Desse modo, realizamos levantamento em livros e artigos de referência no tema, os traduzimos confrontando diferentes versões e analisamos seu conteúdo tendo em vista o anseio de entender e revelar as contribuições matemáticas de Mascheroni. Assim, iniciamos o presente texto com uma apresentação bibliográfica do autor mostrando sua carreira acadêmica dentro e fora da Itália e, na continuidade, apresentamos uma lista de publicações matemáticas de Lorenzo Mascheroni com intenção de divulgar fontes históricas primarias e/ou secundárias, particularmente sobre a matemática do século XVIII, para que outros pesquisadores e/ou professores da educação básica e superior possam utiliza-se de tais obras para elaboração de materiais voltados para o ensino de matemática, a exemplo da intenção original da principal obra de Mascheroni, Problemas para agrimensores com varias soluções, que foi incluída como livro didático utilizado nas aulas do curso de matemática elementar da universidade de Pavia. Como resultado, além de revelar a Geometria feita só com compasso, também chegamos a publicações de Mascheroni relacionadas a outros campos e níveis de ensino, como por exemplo, os textos que caminham por estudos do cálculo diferencial e integral, no estudo do cálculo infinitesimal e ainda temos obras como a coletânea de problemas destinados ao estudo para agrimensores. Portanto, concluímos que sua vasta produção abarca vários aspectos da matemática e sua relação com o ensino que aliás teve reconhecimentos. De fato, temos em uma das obras a apresentação da constante de Euler-Mascheroni, fato que foi reconhecido por sua fundamental contribuição na área da análise matemática, porém seus estudos não se limitam ao estudo do cálculo infinitesimal já que também tratam de Geometria.

\footnotetext{
${ }^{1}$ E-mail: damiaomatematica@ hotmail.com

${ }^{2}$ E-mail: gisellecsousa@hotmail.com
} 
Palavras-chave: Lorenzo Mascheroni; Obras Históricas; Materiais Didáticos; Ensino de Matemática.

\begin{abstract}
In this work we intend to present part of the results of a research, in which we did a historical bibliographical study of the works produced by an eighteenth century Italian mathematician and poet, namely Lorenzo Mascheroni (1750-1800). To do so, we rely on elements of the qualitative research of the bibliographic type, as well as, we use its instruments and procedures. Thus, we carry out a survey of books and articles of reference in the theme, translate them by comparing different versions and analyze their content in view of the yearning to understand and reveal the mathematical contributions of Mascheroni. Thus, we begin the present text with a bibliographical presentation of the author showing his academic career inside and outside Italy and, in the continuity, we present a list of mathematical publications of Lorenzo Mascheroni with the intention of divulging primary and / or secondary historical sources, particularly on the mathematics of the eighteenth century, so that other researchers and / or teachers of basic and higher education can use such works for the elaboration of materials for the teaching of mathematics, such as the original intention of Mascheroni's main work, Problems for surveyors with various solutions, which was included as a textbook used in classes of the elementary mathematics course of the University of Pavia. As a result, in addition to revealing geometry made only with compass, we also come to Mascheroni publications related to other fields and levels of teaching, such as texts that go through studies of differential and integral calculus, in the study of infinitesimal calculus and we still have works like the collection of problems destined to the study for surveyors. Therefore, we conclude that his vast production encompasses several aspects of mathematics and its relation to teaching, which has been recognized. In fact, we have in one of the works the presentation of the constant of Euler-Mascheroni, a fact that was recognized for his fundamental contribution in the area of mathematical analysis, but his studies are not limited to the study of infinitesimal calculus since they also deal with Geometry.
\end{abstract}

Keywords: Lorenzo Mascheroni; Historical Works; Teaching materials; Mathematics Teaching.

\title{
Introdução
}

Com o intuito de apresentar os resultados de uma pesquisa bibliográfica a respeito das produções na área da matemática de Lorenzo Mascheroni (1750 - 1800), para produção e divulgação de futuros materiais voltados ao ensino da matemática, resgatamos a Matemática por ele utilizada. Assim, apresentaremos a biografia deste matemático, usando como fonte as notas bibliográficas presentes na segunda edição de sua obra, $L a$ Geometria del Compasso - A Geometria do Compasso - publicada em 1901 (DELL'OMO, 1901). Além disso, as referidas versões foram traduzidas e seu conteúdo analisado em confronto com outras fontes primárias ou secundárias, incluindo livros de história da matemática, enciclopédias, teses, dissertações, artigos e sites que além de sua 
matemática trazem traços biográficos de Mascheroni. Nessa direção, estruturamos o presente artigo com seções como notas biográficas, Mascheroni e suas produções matemáticas, considerações finais, além da presente introdução.

\section{Notas biográficas de Lorenzo Mascheroni}

Neste momento, passaremos para a apresentação de um matemático e poeta italiano que se tornou conhecido como Lorenzo Mascheroni (ver Imagem 1), cujo nome completo é Lorenzo Mascheroni Dell'Omo. O mesmo nasceu em Castagneta perto de Bérgamo em 13 de Maio de 1750, filho de um jovem casal de fazendeiros, Maria Ceribelli e Paolo Mascheroni dell'Omo. Contudo, Mascheroni não foi educado para torna-se um fazendeiro, seus pais o encaminharam para a escola de seminário de Bérgamo, no qual permaneceu até os seus 18 anos de idade em um regime de estudos que o levou a ser ordenado sacerdote, ainda jovem.

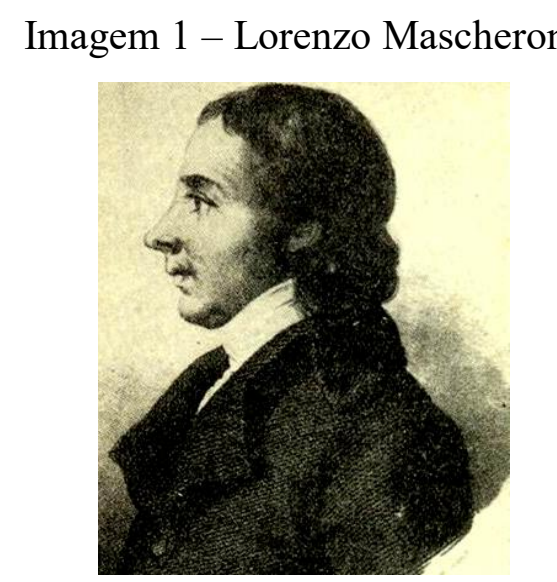

Fonte: Eves (2004)

Diante do material que dispomos para análise, não temos fonte que nos afirme quais os reais motivos que influenciaram Mascheroni a seguir a carreira do sacerdócio, entretanto, como afirmado anteriormente, todos seus estudos ocorreram no seminário de Bérgamo, fato que nos leva indiciar que a vivência dentro do seminário foi de grande influência para tal escolha, já que seu regime de estudo era de internato, passando assim períodos mais longos no seminário que com sua família.

A região de Castagneta, onde Mascheroni nasceu, era um vilarejo que pertencia à cidade de Bérgamo. Em meados do século XVIII, a principal atividade econômica desta região baseava-se em atividades agrárias. Nessa época, tínhamos que o renascimento artístico e científico estava dando passos em rumo às mudanças sociais que contribuíram para unificação das cidades estados, as quais compunham a península itálica. Com essas 
ocorrências, o cenário político não muito estável, no entanto agradável para os que viviam no campo. Contudo, no centro, os moradores estavam passando por um período de paz sem invasões ou disputas feudais que provocassem maiores contendas. Em meio a esse cenário, Mascheroni inicia seus estudos, pesquisas e produção matemática, conforme segue.

\section{Lorenzo Mascheroni e suas produções matemáticas}

Vejamos agora a lista das produções matemáticas de Mascheroni que é apresentada por Fazzari (1901. In DELL'OMO, 1901) nas notas biográficas da segunda edição de A Geometria do Compasso, onde também são indicadas outras fontes consultadas para que fosse possível compor as discussões que aqui seguem sobre as obras de Masceroni. Vale salientar que a produção apresentada está organizada em ordem cronológica indicando assim, ano de publicação, título (original e traduzido) e conteúdo resumido em cada caso.

a) Em 1782, Mascheroni publica: Della più bella proprietà della curva esocrona a direzioni convergenti, ecc - A mais bela propriedade da curva isócrona ${ }^{3} \mathrm{em}$ direções convergentes, etc - Esta é a sua primeira produção matemática que consistia de uma pequena nota publicada em 19 de setembro de 1782, que foi dedicada ao Matemático Aquiles Alessandri $(1665$ - 1751) de Bérgamo. Nela foi demonstrado que a curva isócrona aproxima-se de uma espiral infinita.

b) No mesmo ano, em 1782, foi publicada em panfletos, uma discussão sobre Maniera di misurare l'inclinazione dell'ago calamitato - Maneira de medir a inclinação da agulha magnetizada - A maneira utilizada para medir a inclinação da agulha magnetizada em um local de coordenadas desconhecidas, publicada em Bérgamo, 1782. Anteriormente ao estudo do Mascheroni nesta área, o método utilizado para medir a inclinação magnética era impreciso, porém nas fontes que dispomos não há relatos que exemplifiquem o uso e funcionamento do método desenvolvido por Muscenbroeck (1692 - 1761). É sugerido, nessa publicação de Mascheroni, determinar um ângulo reto no

\footnotetext{
3 "Isócrona ou curva ao longo da qual um corpo cairá com velocidade vertical uniforme (que resulta ser uma parábola semicúbica, com tangente vertical no ponto de cúspide), a determinação da forma assumida por uma haste elástica presa por uma extremidade e suportando um peso na outra, a determinação da forma assumida por uma lâmina retangular flexível com duas bordas opostas mantidas presas horizontalmente e carregada de um líquido pesado e a forma de uma vela retangular enfunada pelo vento." (EVES, 2004, p. 464)
} 
magnético menor, fazendo uso de uma haste vertical de cobre de modo que ela possa facilmente oscilar por diferentes inclinações magnéticas, em diferentes lugares ou no mesmo lugar em diferentes momentos. Em seguida coloca-se a agulha no lugar do meridiano magnético. Assim, ela não permanece horizontal, mas um extremo se inclina em direção ao norte e suas oscilações descrevem uma curva transcendente, pois, Mascheroni mostra que as coordenadas são proporcionais a força magnética. Logo, ele descreve a natureza da curva que apresenta muita analogia com a conchoide ${ }^{4}$ de Nicomedes século II a.C e com a epicicloide ${ }^{5}$ de Giovanni Bernoulli (1667 - 1705).

c) Em 1784 Mascheroni publica: Sulle curve Che servono a delineare Le are ineguali degli antichi nelle superfície piane - As curvas usadas para definir horas desiguais na antiga superficie plana - Esta obra foi uma breve nota publicada pela primeira vez no volume VII dos folhetos céticos, sobre as ciências e as artes em Milão no ano de 1784. Depois, foi republicada na segunda edição da obra Nuovo ricerche sull'equilibrio delle volte, em 1829 - Uma nova pesquisa sobre o equilíbrio do tempo. Neste folheto Mascheroni provou ser exata a observação que já tinha sido indicada por Scipione Dehe (s/d), que as horas desiguais dos judeus e dos romanos não devem ser marcadas por linhas retas em superfícies planas. Para ilustrar, grava em um disco de bronze a meridiana, no qual são traçadas as linhas que definem as horas dos diferentes sistemas antigos. Esse instrumento, até a data da publicação da segunda edição de $A$ Geometria do Compasso, pertencia à biblioteca pública de Bérgamo, ou seja, até 1901.

d) Em 1785 Mascheroni publica a quarta obra matemática: Nueve recerche sull'equilibrio delle volte. Segundo Fazzari (1901. In DELL'OMO, 1901) esse trabalho - que é geralmente considerado o maior e para o qual o inteligente autor com uma reputação de hábil geômetra viera a produzir, - foi impresso pela primeira vez em Bérgamo em 1785 (in $-4^{\circ}$, con 13 tav.). Em seguida, a obra foi reimpressa com o acréscimo e elogios de Masch. Ferdinando Landi (1778 - 1853) em Milão em 1829 (in $16^{\circ}$, con 5 tav.). Nessa obra Mascheroni mostra não ter um domínio apenas da Mecânica e da Geometria, mas também do Cálculo Infinitesimal, um assunto novo e que havia sido

\footnotetext{
4 "Seja $c$ uma curva dada e $O$ um dado ponto fixo. Sobre o raio vetor $O P$ de $O$ a um ponto $P$ de $c$ marque $P Q= \pm k$, em que $k$ é uma constante. Então o lugar dos pontos $Q$ chama-se conchoide de $c$ para o pólo $O$ e a constante $k$. A curva completa consiste de dois ramos, um corresponde a $P Q=+k$ eo outro $P Q=-k$. Se $c$ é uma reta e $O$ um ponto fora de $c$, obtém-se um conchoide de Nicomedes." (EVES, 2004, p. 152)

5 "Uma epicicloide é uma curva descrita por um ponto da circunferência de um círculo que rola externamente sobre um círculo fixo tomado como base." (EVES, 2004, p. 411)
} 
tratado por poucos matemáticos qualificados, como Giacomo Bernaulli (1654-1705), Gregory (1659- 1708), Hyre (s/d) e Lorgna (1735-1796). No entanto, sua obra não perde a originalidade, pois ele adiciona uma nova e importante verdade sobre o assunto, uma de suas contribuições ficou conhecida como a constante de Euler-Mascheroni. Essa obra também recebe elogios de matemáticos da Bossut scriveagli, fazendo a seguinte declaração: "Você tratou nesta obra um grande número de questões interessantíssimas e muito úteis; e você mostrou grande sagacidade em manipular a análise ${ }^{6}[. .$.$] ” (FAZZARI.$ 1901, In DELL'OMO. 1901, p. VII, tradução nossa).

Na segunda edição italiana do curso de matemática da Bossut, traduzida pelo padre Andrea Mazzone (s/d), publicada em Pavia em 1787, Mascheroni compõe um livro didático que foi usado em muitas das escolas italianas, inclusive por Mascheroni para suas aulas de Matemática Elementar na Universidade de Pavia.

Em uma segunda edição desse curso, foi identificada nos apêndices, uma das produções de Mascheroni metodo di missirare ipolegoni Poligoni piani - método de medição de polígonos planos - que no mesmo ano foi publicada separadamente em Pavia. Nessa publicação Mascheroni ensina como medir um polígono côncavo ou convexo qualquer, sem que seja necessário fazer uso da resolução do polígono em triângulos, determinando alguns elementos de outros lados. Ao determiná-los encontram-se as descrições de um destino pelo qual não só encontra-se a medida de um ângulo, como também seu seno e cosseno. Nesse livreto são identificadas as harmonias entre a clareza e a qualidade de seus escritos, bem como em todo o resto de suas obras. Assim notamos que seus estudos literários influenciavam no modo de escrever suas obras matemáticas. Diante da importância didática que tal obra apresenta, Huilier (s/d) insere os problemas e as soluções deste pequeno trabalho em sua obra polygonométrie, publicada dois anos depois em Genebra no ano de 1789, sem fazer qualquer menção ao trabalho do matemático italiano. Perante esta indesejável situação, tudo que o Mascheroni nobremente fez no prefácio de seus Problemi per gli agrimensari - Problemas para os agrimensores - foi escrever algumas palavras a respeito de tal publicação, afirmando ter tomado conhecimento da publicação de Huilier em que seu método estava contido, bem como todos os problemas, acrescentados de soluções analíticas, afirma ainda ter ficado surpreendido que tal matemático tenha seguido os mesmos caminhos e dados os mesmos

\footnotetext{
${ }^{6}$ Vous avez traitè dans cet ouvrage un grand nombre de questions très-intèressantes et très-utiles; et vous y montrez une grande sagacitè á manier l'analyse.
} 
passos, sendo estes os que ele já tinha percorrido dois anos antes, acrescenta ainda que, apesar de tais coincidências, o livro do Huilier merece seus créditos, pois apresenta novas provas geométricas. Tal fato revela que Mascheroni não era arrogante, pois tinha respeito pelos demais. De fato, mesmo na situação descrita anteriormente, soube manter um bom relacionamento com o autor que tinha dado algumas contribuições a uma de suas obras e republicada sem fazer qualquer menção da obra original de Mascheroni.

Mascheroni, no início de seus estudos em Matemática, já tinha se deparado com Cálculo Diferencial e Integral, entretanto, tinha realizado apenas uma publicação nesta área, até que ao estudar as produções de Leonhard Euler (1707-1783), fez as seguintes publicações.

e) Em 1790 Mascheroni publica: Ad notationes ad calculum integralem Euleri in quibus nonnulla problemata ab Eulero propesita resolventur, etc. (Ticini, 1790).

f) Em 1792 Mascheroni publica: Adnotationum ad calculum integralem Euleri in quibus nonnullae formulae ab Eulero propositae plenius evolvuntur. Pars altera, etc. (Ticini, 1792).

As duas publicações citadas anteriormente dizem respeito aos estudos do Mascheroni na área do Cálculo Integral. Nestes volumes, o autor, aumentando a nitidez de seu intelecto, tem o propósito de resolver várias questões propostas pelos maiores matemáticos do século XVIII e em especial é notável que suas pesquisas giram em torno da determinação do logaritmo integrante, ou seja, a integral do logaritmo.

g) Em 1791 Mascheroni publica: Adnotationes Mascheroni in $R$. Archigymnasio Ticinensi mathen. Prof. No final do segundo volume da segunda edição da obra de Wolfio, Elementa Matheseos universae, publicada em 1791 na comunidade de Verona, Itália. Essa obra de Mascheroni consistia numa lista de quinze notas, todas referentes ao trabalho mecânico estático, no qual são abordados problemas de mecânica referente aos estudos de estática.

h) Em 1793 Mascheroni publica a sua nona obra tratando do conjunto de problemas intitulado por Problemi per gli Agrimensori con varie soluzioni - Problemas para agrimessores com várias soluções. Este trabalho vem fornecer contribuições para a Geometria della Riga - Geometria da Régua. Foi publicada pela vez primeira em Pavia em 1793, e também republicada em Milão em 1832 com o título: problemi di geometria colle dimostrazioni aggiunte dal cittadino Sacchi, capitano in 2. artiglieria, ecc. (in - $8^{o}$ con 4 tav). Nesta segunda edição (a de Milão) realizada por Sacchi (s/d), foram acrescidas 
demonstrações que tinham sido recomendadas pelo próprio Mascheroni. Este mesmo trabalho foi publicado em Milão em 1832, com o título: Problemas de geometria com demonstrações do capitão Sacchi, edição enriquecida pela adição de alguns problemas derivados de uma cópia da primeira edição a notada pelo autor (in-16 ${ }^{\circ}$, con 5 tav.), no formato de um livro recomendado para os estudantes das escolas secundárias. A mesma aparece editada em francês por um coautor anônimo, em Paris em 1803. A obra é composta por cinco livros, no qual o primeiro versa sobre Aritmética em que o autor aborda dois métodos para calcular a distância entre dois pontos inacessíveis; no segundo livro há um tratamento sobre dois métodos para medição de ângulos e para traçar retas ortogonais sem o auxílio de equipamentos ou grafômetros ${ }^{7}$; o terceiro é composto por problemas sobre medição das superfícies planas e curvas; o quarto livro discute novamente sobre sua polygométria, já publicada em um panfleto, que faz menção principalmente para generalização das fórmulas que podem ser usadas em qualquer espaço para medir linhas retas; no quinto livro aborda sobre o método de medição para poliedros.

i) Em 1795 Mascheroni publica a décima obra que ocorreu no segundo volume do jornal de Física e Medicina, de L. Brugnatelli publicada em Pavia em 1795. Trata-se de uma carta intitulada lettera all'illustre signor don Annibale Beccaria patrizio milanese, con alcuni problemi geometri sciolti col cerchio senza la regola. Esta carta continha uma lista de problemas geométricos a respeito do círculo, um dos problemas abordados é o da divisão da circunferência em vinte e quatro partes iguais.

j) Em 1797 Mascheroni publica a décima primeira obra que é a intitulada por La Geometria del compasso. A referida obra aborda problemas de construções geométricas, que são realizadas apenas com auxílio do compasso. $\mathrm{O}$ autor as resolve mostrando as construções com seus passos e finaliza com as demonstrações algébricas. Ao todo constitui de uma coleção de 12 livros, a exemplo da obra de Euclides que é composta por 13 livros. Nos livros da obra de Mascheroni são abordados problemas variados a respeito das construções geométricas em que se utiliza apenas o instrumento compasso.

k) Em 1798 Mascheroni publica: Notizie generali del nuovo sistema dei perie misure defotti dalla grandezza Terra, publicada em Milão, 1798 (in - 8). Esta obra trata

\footnotetext{
${ }^{7}$ Instrumento utilizado no levantamento de planos para a medição dos ângulos de um terreno.
} 
de seus estudos na comissão francesa para determinar o novo sistema de pesos e medidas. Entretanto, já em 1780 o Cesare Beccaria (1738 - 1794) apresentou ao magistrado da câmara de Milão uma proposta de uniformidade das medidas de comprimentos propondo dividir as medidas em frações decimais, mas sua proposta foi deixada no esquecimento até a publicação do Mascheroni, que estimula o corpo legislativo da República Cisalpina, em 11 de março de 1798, a instituir uma comissão responsável para determinar um sistema de pesos, medidas e moeda, semelhante ao da República Francesa. Mascheroni, que continua entre os comissários, fica responsável por tornar agradável ao público o novo sistema, explicando todas as utilidades praticadas.

Para finalizarmos nosso recorte sobre suas publicações ligadas à matemática, temos que no nono volume das memórias de Matemática e Física da sociedade Italiana da ciência, de 1802, encontra-se inserido uma obra póstuma de Mascheroni com o título Spiegazione popolare della maniera con la quale si regola l'onno sestile o intercalare ed il cominciamento dell'anno Republicano, que tinha sido enviada em 13 de Abril de 1799 para Serbelloni (s/d), embaixador da república francesa na República Cisalpina.

\section{Considerações finais}

Após termos discorrida sobre a lista de obras publicadas por Lorenzo Mascheroni, fica notório que tal conjunto de materiais tem potencial significativo para o desenvolvimento de estudos na área da Educação Matemática, e, buscando meios de divulgação, produzimos este texto com a principal finalidade de mostrarmos a comunidade acadêmica uma rica fonte capaz de embasar pesquisas que estejam voltadas para produção de materiais didáticos em que a história da matemática seja agente da construção do saber matemático educacional. Como exemplo de proposta didática usando tal material, temos o produto educacional da dissertação intitulada A Geometria do Compasso (1797) de Mascheroni (1750 - 1800) em Atividades com Geogebra de autoria de Oliveira (2014) e orientação de Sousa.

\section{Referências}

DELL'OMO, Lorenzo Mascheroni. La Geometria del Compasso. 2 ed. Palermo: era nova, 1901.

1797.

La Geometria del Compasso. ed. Pavia ano V della Repubblica Francesa, 
José Damião Souza de Oliveira e Giselle Costa de Sousa

As produções matemáticas de Lorenzo Mascheroni como fonte para produção de materiais educacionais

Géométrie du Compas. Tradução: A. M. Carette ed. Paris ano VI della

Repubblica Francesa, 1798.

EVES, Howard. Introdução à História da Matemática. Tradução: Hygino $\mathrm{H}$. Domingues. Campinas: Editora da UNICAMP, 2004.

OLIVEIRA. José Damião Souza de. A geometria do compasso (1797) de Mascheroni (1750 - 1800) em atividades com GeoGebra. 2014. 221f. Dissertação (Mestrado) Universidade Federal do Rio Grande do Norte, Natal, 2014. 